\title{
Characteristics and outcomes of 627044 COVID-19 patients living with and without obesity in the United States, Spain, and the United Kingdom
}

\author{
Martina Recalde ${ }^{1,2,36}$, Elena Roel (D) ${ }^{1,2,36}$, Andrea Pistillo (D) ${ }^{1}$, Anthony G. Sena (D) ${ }^{3,4}$, Albert Prats-Uribe (iD) ${ }^{5}$, \\ Waheed-Ul-Rahman Ahmed ${ }^{6,7}$, Heba Alghoul ${ }^{8}$, Thamir M. Alshammari (iD) ${ }^{9}$, Osaid Alser ${ }^{10}$, Carlos Areia (iD ${ }^{11}$, Edward Burn ${ }^{1,5}$, \\ Paula Casajust ${ }^{12}$, Dalia Dawoud ${ }^{13}$, Scott L. DuVall ${ }^{14,15}$, Thomas Falconer ${ }^{16}$, Sergio Fernández-Bertolín ${ }^{1}$, Asieh Golozar ${ }^{17,18}$, \\ Mengchun Gong ${ }^{19}$, Lana Yin Hui Lai ${ }^{20}$, Jennifer C. E. Lane ${ }^{6}$, Kristine E. Lynch ${ }^{14,15}$, Michael E. Matheny ${ }^{21,22}$, Paras P. Mehta ${ }^{23}$, \\ Daniel R. Morales ${ }^{24}$, Karthik Natarjan ${ }^{16,25}$, Fredrik Nyberg ${ }^{26}$, Jose D. Posada ${ }^{27}$, Christian G. Reich ${ }^{28}$, Peter R. Rijnbeek ${ }^{4}$, Lisa M. Schilling ${ }^{29}$, \\ Karishma Shah ${ }^{6}$, Nigam H. Shah ${ }^{27}$, Vignesh Subbian ${ }^{30}$, Lin Zhang ${ }^{31,32}$, Hong Zhu ${ }^{33,34}$, Patrick Ryan ${ }^{3,16}$, Daniel Prieto-Alhambra, \\ Kristin Kostka ${ }^{28,35,37}$ and Talita Duarte-Salles ${ }^{1,37 凶}$
}

(c) The Author(s) 2021

BACKGROUND: A detailed characterization of patients with COVID-19 living with obesity has not yet been undertaken. We aimed to describe and compare the demographics, medical conditions, and outcomes of COVID-19 patients living with obesity (PLWO) to those of patients living without obesity.

METHODS: We conducted a cohort study based on outpatient/inpatient care and claims data from January to June 2020 from Spain, the UK, and the US. We used six databases standardized to the OMOP common data model. We defined two non-mutually exclusive cohorts of patients diagnosed and/or hospitalized with COVID-19; patients were followed from index date to 30 days or death. We report the frequency of demographics, prior medical conditions, and 30-days outcomes (hospitalization, events, and death) by obesity status.

RESULTS: We included 627044 (Spain: 122 058, UK: 2336, and US: 502 650) diagnosed and 160013 (Spain: 18 197, US: 141 816) hospitalized patients with COVID-19. The prevalence of obesity was higher among patients hospitalized (39.9\%, 95\%Cl: 39.8-40.0) than among those diagnosed with COVID-19 (33.1\%; 95\%Cl: 33.0-33.2). In both cohorts, PLWO were more often female. Hospitalized PLWO were younger than patients without obesity. Overall, COVID-19 PLWO were more likely to have prior medical conditions, present with cardiovascular and respiratory events during hospitalization, or require intensive services compared to COVID-19 patients without obesity.

CONCLUSION: We show that PLWO differ from patients without obesity in a wide range of medical conditions and present with more severe forms of COVID-19, with higher hospitalization rates and intensive services requirements. These findings can help guiding preventive strategies of COVID-19 infection and complications and generating hypotheses for causal inference studies.

International Journal of Obesity (2021) 45:2347-2357; https://doi.org/10.1038/s41366-021-00893-4

\section{INTRODUCTION}

Obesity is associated with increased mortality and is a well-known risk factor for chronic conditions, such as diabetes, hypertension, cardiovascular disease, and cancer [1,2]. Due to its proinflammatory state that impairs the immune response, obesity has also been related to an increased risk of viral infections [3]. The novel coronavirus disease 2019 (COVID-19), caused by the severe acute respiratory syndrome coronavirus 2 (SARS-CoV-2), emerged in December 2019 in Wuhan, China, and rapidly spread around the world [4]. This new virus causes a respiratory tract infection with clinical manifestations ranging from asymptomatic/mild symptoms to severe illness requiring intensive services. Partly due to its similarities with other viral infections such as seasonal influenza or H1N1, people with obesity were soon labeled as "at-risk" individuals [5]. Since obesity is a worldwide public health priority, granular information on patients with COVID-19 and obesity is needed to guide preventive strategies as well as to generate hypotheses for etiological studies [6].

A review and meta-analysis of 75 studies reported that obesity is a risk factor for testing positive for SARS-CoV-2, for severe COVID-19 and for COVID-19 related mortality [7]. While undoubtedly relevant to the field, these studies mainly focused on exploring multiple risk factors related to COVID-19 and thus did not offer a detailed characterization of patients with COVID-19

A full list of author affiliations appears at the end of the paper 
living with obesity. For instance, an exhaustive description of the medical conditions and COVID-19 related outcomes, such as thromboembolic events, among these patients is lacking. Other current limitations include the susceptibility to collider bias of studies reporting "risk factors" of COVID-19 infection and progression due to sampling mechanisms (e.g., subsamples of tested or hospitalized populations) [8]. A large characterization study focussing exclusively on patients with COVID-19 living with obesity using real-world data from different health settings and countries could address the limitations of the previous evidence.

In this study, we aimed to describe and compare the demographics, medical conditions, and outcomes of COVID-19 patients living with obesity (PLWO) to those of COVID-19 patients living without obesity, in inpatient or outpatient settings.

\section{METHODS}

\section{Study design, setting, and data sources}

We conducted a multinational cohort study using routinely collected healthcare data from January to June 2020 from Spain, the United Kingdom (UK), and the United States (US). This study was part of the "Characterizing Health Associated Risks, and Your Baseline Disease In SARS-COV-2 (CHARYBDIS)" study (protocol available for download at https://www.ohdsi.org/wp-content/uploads/2020/07/Protocol_COVID19-Charybdis-Characterisation_V5.docx) designed by the Observational Health Data Sciences and Informatics (OHDSI) community. All data were standardized to the Observational Medical Outcomes Partnership (OMOP) Common Data Model (CDM) [9]. The OHDSI network maintains the OMOP-CDM, along with a wide range of tools developed by its members to facilitate analyses of mapped data [10]. Data results for this study were extracted on July, 16th, 2020 .

We included primary, outpatient and inpatient care data from electronic health records (EHRs) and health insurance claims data from six databases. Data from Spain included the Information System for Research in Primary Care (SIDIAP), which includes primary linked to inpatient care data covering approximately $80 \%$ of the population in Catalonia, Spain [11]. The UK data covered the Clinical Practice Research Datalink (CPRD), with patients from over 600 general practices in the UK [12]. Data from the US included: Columbia University Irving Medical Center (CUIMC), covering New York-Presbyterian Hospital and its affiliated physician practices; IQVIA Open Claims, which are pre-adjudicated claims collected from office-based physicians and specialists covering over 300 million lives ( $80 \%$ of the US population); the Stanford Medicine Research Data Repository (STARROMOP), with data from Stanford Health Care [13], and the United States Department of Veterans Affairs (VA-OMOP), covering the national Department of Veterans Affairs health care system which serves more than 9 million enrolled Veterans (of whom 93\% are male). A more detailed description of the included data sources is available in Supplementary Appendix 1.

\section{Study participants}

We included two non-mutually exclusive cohorts of patients: (1) all patients diagnosed with COVID-19 (clinical diagnosis and/or positive test for SARSCoV-2), and (2) all patients hospitalized with a COVID-19 diagnosis. We considered clinical diagnoses in the definition of COVID-19 cases due to testing restrictions during the first months of the pandemic (e.g., in Spain) [14]. The diagnostic codes used are described in Supplementary Appendix 2. Patients hospitalized with COVID-19 were identified as those having a hospitalization episode along with a clinical diagnosis or positive SARSCoV-2 test within a time window from 21 days prior to admission up to the end of their hospitalization. We chose this time window to include patients with a diagnosis prior to hospitalization and to allow for a record delay in test results or diagnoses [15]. We included individuals with at least one year of observation time prior to the index date to capture observed baseline characteristics. In the diagnosed cohort, the index date was defined as the date of the COVID-19 clinical diagnosis or the earliest test day registered within seven days of a first positive test, whichever occurred first. In the hospitalized cohort, the index date was the day of hospitalization. Patients were followed from the index date to the earliest of death, end of the observation period, or 30 days [16].

Both the diagnosed and hospitalized COVID-19 cohorts were stratified by obesity status: PLWO vs patients living without obesity (from now on, referred to as patients without obesity). Obesity was defined as having an ever-recorded obesity diagnosis (Supplementary Appendix 3) and/or a body mass index (BMI) measurement between 30 and $60 \mathrm{~kg} / \mathrm{m}^{2}$ and/or a bodyweight measurement between 120 and $200 \mathrm{~kg}$ prior or at index date. We included upper cut-off thresholds to discard implausible observations. Patients without obesity were those who did not fulfill the obesity definition.

\section{Baseline characteristics and outcomes of interest}

Demographics (sex and age) were obtained at the index date. More than 15000 medical conditions from up to one year prior to the index date were identified based on the Systematized Nomenclature of Medicine (SNOMED) hierarchy, with all descendant codes included [15]. Specific definitions for comorbidities of particular interest were created; the detailed definitions of these variables can be consulted in Supplementary Appendix 3. We reported here a list of key comorbidities based on their prevalence in the cohorts of the participating sites, as well as on their clinical relevance to obesity and the COVID-19 research field [17].

Our 30-day outcomes of interest for the diagnosed cohort were hospitalization and fatality. For the hospitalized cohort, the 30-days outcomes were a requirement of intensive services (IS) (identified by a recorded mechanical ventilation and/or a tracheostomy and/or extracorporeal membrane oxygenation procedure), respiratory, cardiovascular, thromboembolic, and other events and fatality.

\section{Data analysis}

We described the number of patients included and the prevalence of obesity in each database as well as the demographics, comorbidities, and outcomes as proportions (calculated by the number of persons within a given category, divided by the total number of persons) with their respective $95 \%$ confidence intervals (Cls) for each database, by obesity status. To calculate these proportions in each database, we established a minimum count required (of five individuals), to minimize the risk of reidentification of patients. To compare medical conditions across groups, we calculated standardized mean differences (SMDs) [18], which we summarized in Manhattan-style plots. The SMD can be used to compare the prevalence of a dichotomous variable between two groups and is independent of sample size [19]. A $|S M D|>0.1$ indicates a meaningful difference in the prevalence of a given condition; in the context of this study, a SMD $>0.1$ indicates a higher prevalence in PLWO, whereas a $\mathrm{SMD}<-0.1$ indicates a higher prevalence among patients without obesity. This study was descriptive by nature and, therefore, statistical modeling was out of scope. Differences across the groups compared should not be interpreted as causal effects.

To ensure data privacy at all times, we employed a federated analysis approach [16]. Following a pre-specified analysis plan, a common analytical code for the whole CHARYBDIS study was developed for the OHDSI Methods library, available at https://github.com/ohdsi-studies/ Covid19CharacterizationCharybdis, and was run locally in each database. Individual-level data remained within host institutions and only aggregate results from each database were provided to the research team and publicly shared. All the results reported in this paper and additional data are available for consultation at a dynamic and interactive website, which changes over time as new databases are added and/or results are updated to CHARYBDIS (https://data.ohdsi.org/ Covid19CharacterizationCharybdis/).

We used $\mathrm{R}$ version 3.6 for data visualization. All the data partners obtained Institutional Review Board (IRB) approval or exemption to conduct this descriptive study.

\section{RESULTS}

\section{Prevalence of obesity}

We included 627044 diagnosed and 160013 hospitalized patients with COVID-19 (Table 1). The diagnosed cohort consisted of 122 058 patients from Spain (SIDIAP), 2336 from the UK (CPRD), and 502650 from the US (CUIMC: 8519; IQVIA-OpenClaims: 466 191; STARR-OMOP: 3328; VA-OMOP: 24 612). The hospitalized cohort included 18197 patients from Spain (SIDIAP) and 141816 from the US (CUIMC: 2600; IQVIA-OpenClaims: 133 091; STARR-OMOP: 615; VA-OMOP: 5510). Among diagnosed and hospitalized patients, 207859 (33.1\%; 95\%Cl: 33.0-33.2) and 63866 (39.9\%, 95\%Cl: 39.8 $-40.0)$ had obesity, respectively. In all databases, the prevalence 


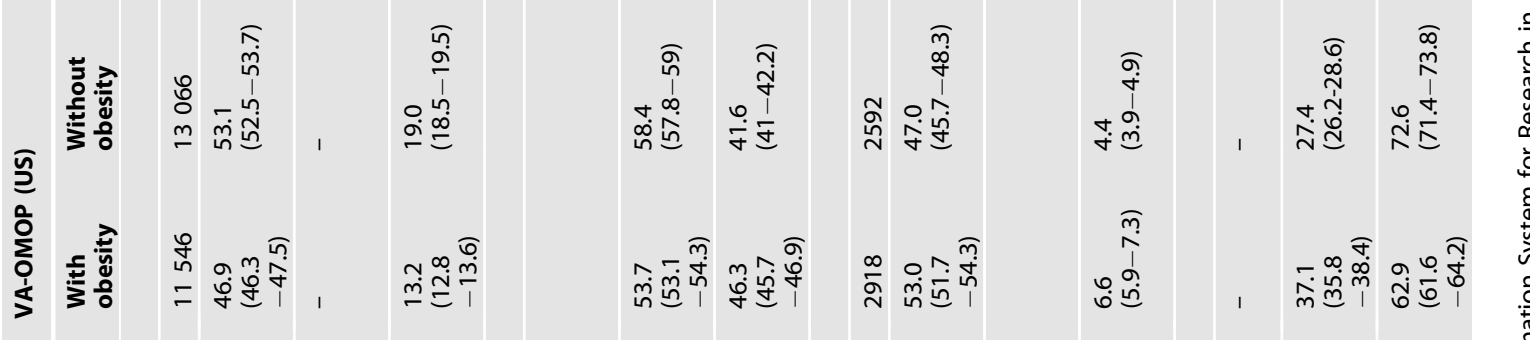

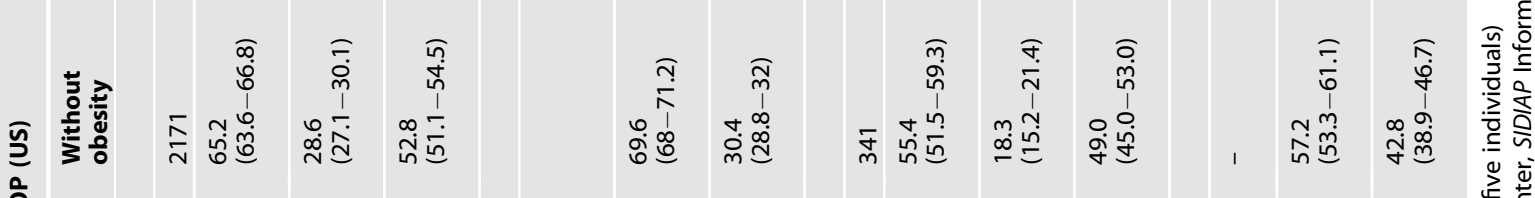

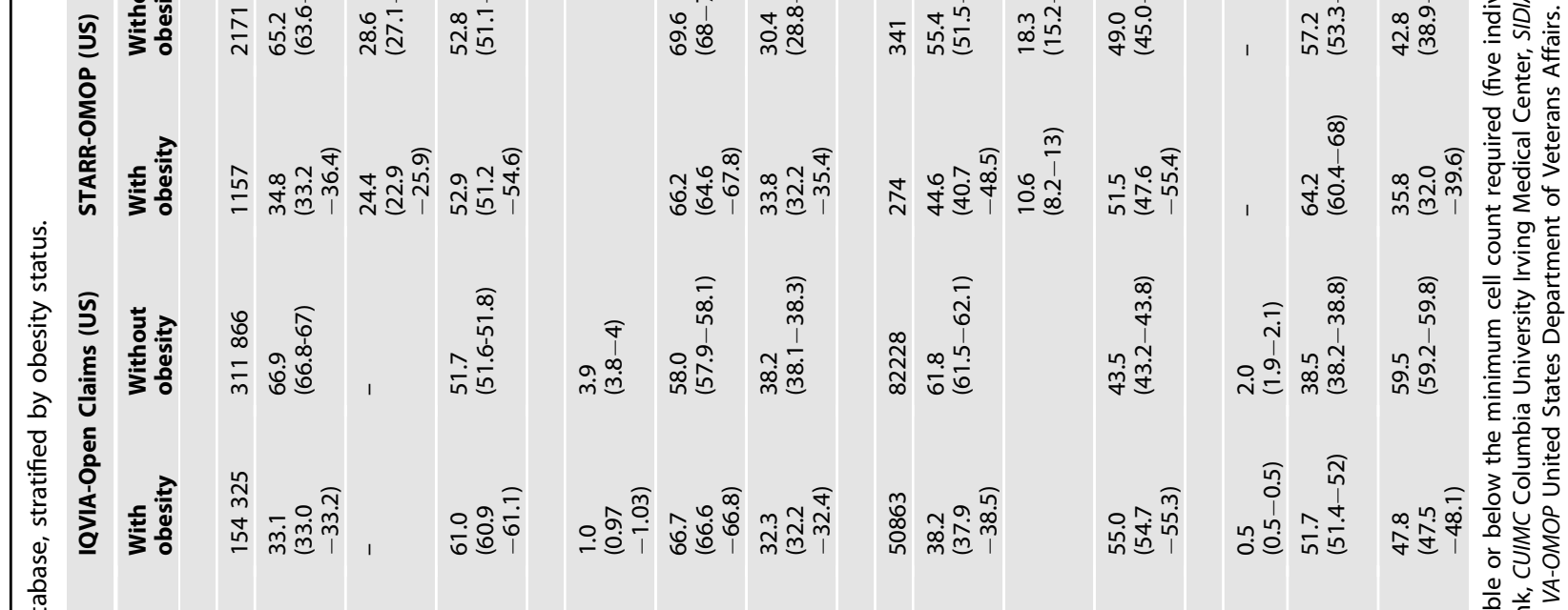

密

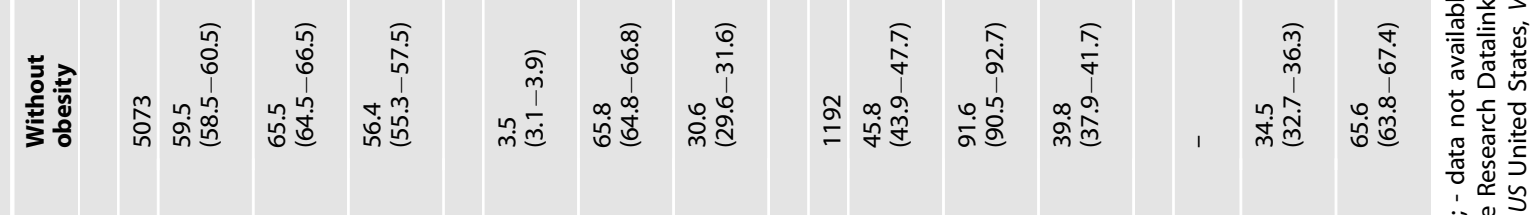

氙

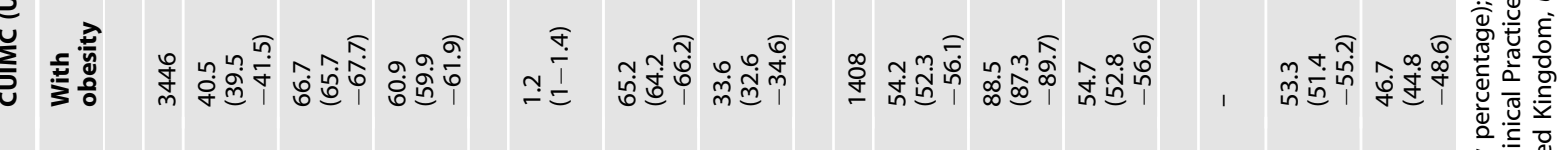

高高

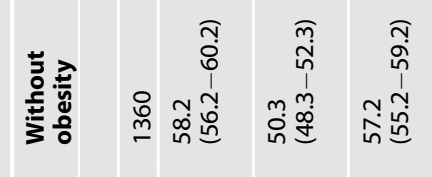

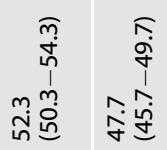

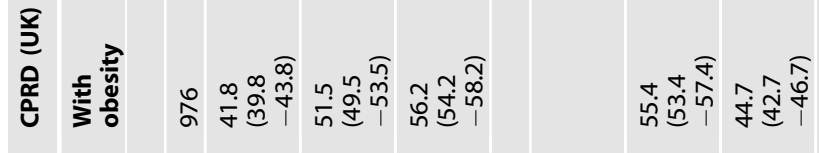

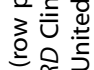

氨高

要

$\overline{\overline{\mathrm{T}}} \stackrel{\mathrm{g}}{\mathrm{o}}$

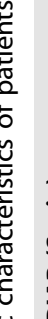

ํํำ

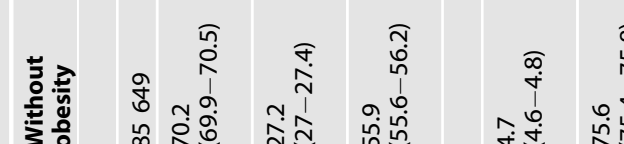

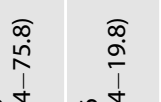

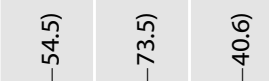

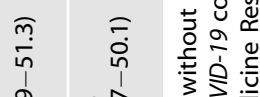

要

垔

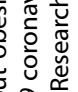

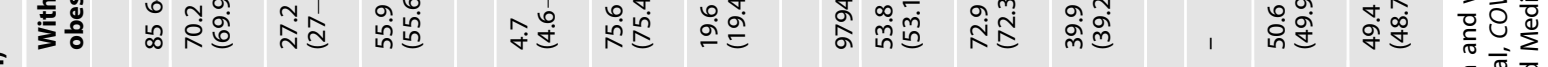

这

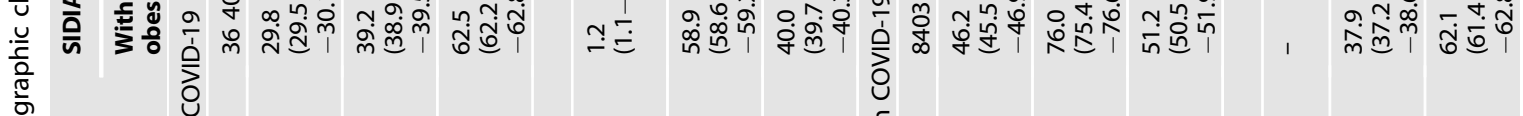

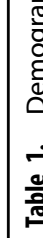

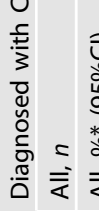

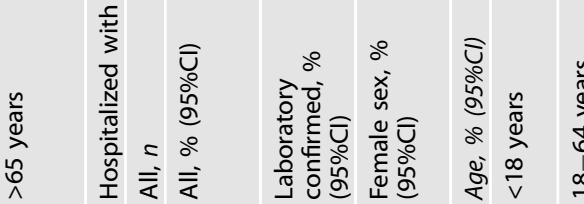

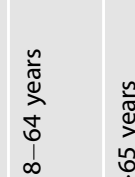
to

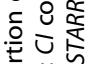

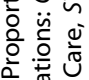

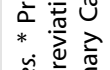
$\sum_{2}^{\circ} \frac{0}{2} \frac{\xi}{2}$ 

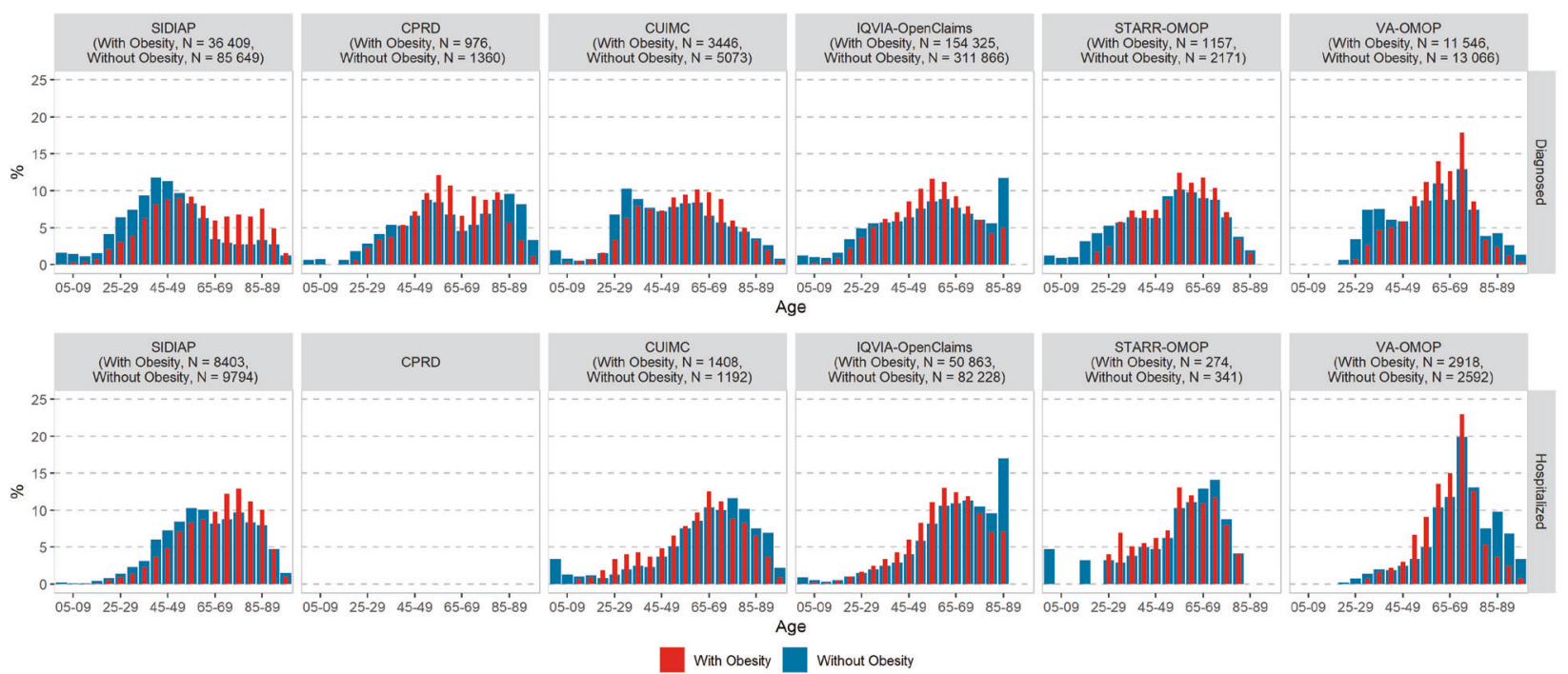

Fig. 1 Distribution of age among patients living with and without obesity in each database, stratified by COVID-19 cohort type (diagnosed and hospitalized). CPRD Clinical Practice Research Datalink, COVID-19 coronavirus disease 2019, CUIMC Columbia University Irving Medical Center, SIDIAP Information System for Research in Primary Care, STARR-OMOP Stanford Medicine Research Data Repository, VAOMOP United States Department of Veterans Affairs.

of obesity was lower among diagnosed patients than among those hospitalized, with differences ranging from 5 (IQVIA-OpenClaims) to $16 \%$ (SIDIAP).

\section{Baseline demographics}

The sex distribution (proportions and $95 \% \mathrm{Cls}$ ) of the patients are reported in Table 1. Aside from VA-OMOP, in the diagnosed cohort, patients with and without obesity were mostly female. The proportion of females was higher among PLWO compared to patients without obesity in SIDIAP (63\% vs $56 \%$ ), CUIMC (61\% vs $56 \%)$, and IQVIA-OpenClaims (61\% vs $52 \%$ ), while in VA-OMOP the opposite was observed (13\% vs $19 \%)$. No differences were observed in CPRD and STARR-OMOP. In the hospitalized cohort, patients without obesity were predominantly male (female ranged from 40 to $49 \%$, VA-OMOP: $4 \%$ ) but PLWO still were more commonly female in all databases aside from VA-OMOP (range: $51-55 \%$, VA-OMOP: $7 \%$ ). Differences in the proportion of females between PLWO and patients without obesity ranged from 3 (VA-OMOP) to 15\% (CUIMC).

The age distribution in each database is summarized in Table 1 with proportions and their respective $95 \% \mathrm{Cls}$ and in Fig. 1 with histograms. In the diagnosed cohort, PLWO were slightly older than those without obesity (i.e., the age distribution for PLWO was slightly skewed to the left compared to patients without obesity). This was particularly marked in SIDIAP, where $40 \%$ of the PLWO were aged above 65 years and only $20 \%$ were so without obesity. Hospitalized patients were older than those diagnosed. In the hospitalized cohorts, PLWO were fairly consistently younger than those without obesity (except for SIDIAP). The proportion of patients aged above 65 ranged from 36 to $63 \%$ for PLWO and from 43 to $73 \%$ for those without obesity.

\section{Baseline medical conditions}

We compared baseline medical conditions of PLWO to those of patients without obesity in the diagnosed and hospitalized cohorts using SMDs, which are summarized in Fig. 2. We depicted the SMDs of 485 (CPRD) to 5050 (VA-OMOP) medical conditions in the diagnosed cohort, and 529 (STARR-OMOP) to 5240 (IQVIA-OpenClaims) in the hospitalized cohort. In both cohorts, medical conditions were largely more frequent among PLWO than patients without obesity.

The distribution of the selected key comorbidities is shown in Fig. 3, and the proportions with their respective $95 \%$ Cls and SMDs between PLWO and patients without obesity are available in Supplementary Appendices 4 and 5. In the diagnosed cohorts, PLWO consistently had a higher prevalence of comorbidities compared to those without obesity; these differences were meaningful (i.e., with a SMD $>0.1$, which indicates a meaningfully higher prevalence among PLWO) for the majority of comorbidities across databases. For example, while the prevalence of hypertension for PLWO ranged from 30 to $32 \%$ in Europe (SIDIAP and CPRD) and from 55 to $81 \%$ in the US, in those without obesity it ranged from 12 to $16 \%$ and from 26 to $53 \%$, respectively. The SMD for hypertension was above 0.1 in all databases. As in the diagnosed cohort, PLWO hospitalized with COVID-19 had a higher prevalence of comorbidities than those without obesity, and these differences were meaningful for the majority of comorbidities. However, the differences between groups were less obvious. For example, heart disease differed by $20 \%$ among those diagnosed in VA-OMOP (PLWO: 60\%, without obesity: $40 \%$ ) and by $9 \%$ among those hospitalized (PLWO: 74\%, without obesity: $65 \%$ ); although the SMD was still above 0.1 in all databases.

\section{0-day outcomes of interest}

The distribution of 30-days outcomes is shown in Fig. 4, the proportions with their respective $95 \% \mathrm{Cl}$ and SMDs between PLWO and patients without obesity are available in Table 2 . In the diagnosed cohorts, hospitalization rates were higher among PLWO than among those without obesity in all databases. For example, in SIDIAP the proportion of patients hospitalized was $20 \%$ for PLWO and $10 \%$ for patients without obesity. However, these differences were meaningful $(S M D>0.1)$ only in three databases: SIDIAP, CUIMC, and STARR-OMOP. In PLWO, fatality ranged from 5 to $12 \%$ and was higher than in patients without obesity in SIDIAP and CUIMC ( $7 \%$ vs $3 \%$ and $8 \%$ vs $5 \%$, respectively), while in CPRD and VA-OMOP it was similar in both groups. SIDIAP was the only database with a meaningful difference in the proportion of fatality.

Overall, in the hospitalized cohort, PLWO more frequently had adverse events occurring in the 30 days after the index date than patients without obesity. For example, PLWO required IS and presented with ARDS more frequently than patients without obesity in the largest databases: IQVIA-OpenClaims (IS: $13 \%$ vs $10 \%$; ARDS: $35 \%$ vs $31 \%$ ) and VA-OMOP (IS: $22 \%$ vs $15 \% ; 46 \%$ vs $41 \%)$, whereas in CUIMC and STARR-OMOP percentages were similar. VA-OMOP was the only database with a meaningful 


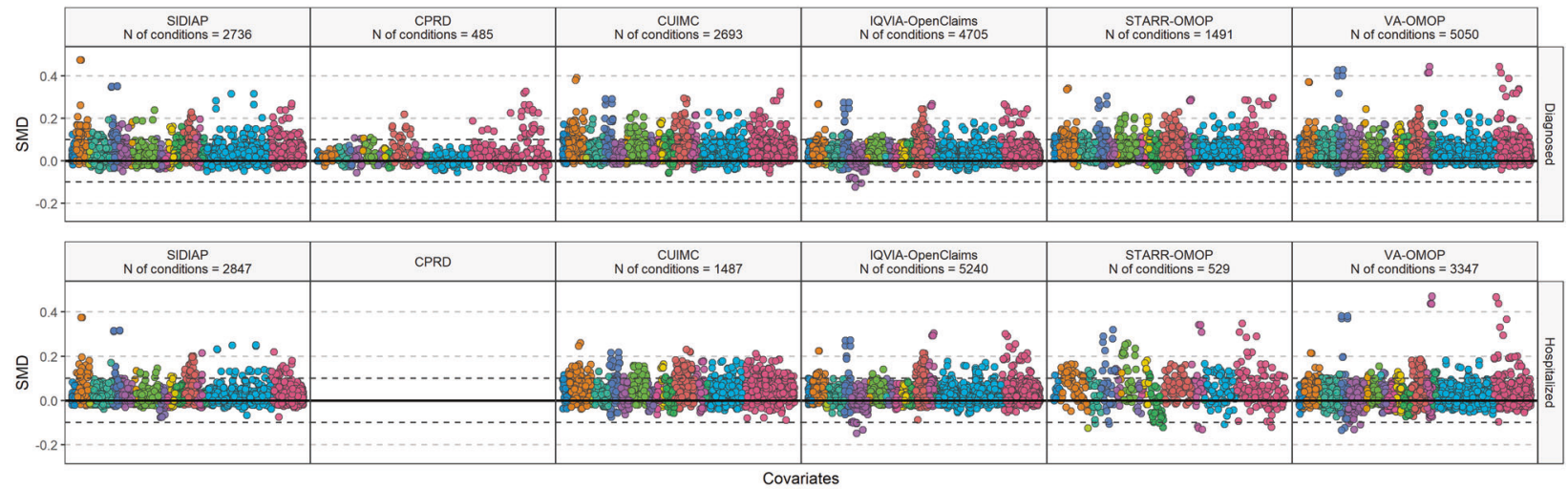

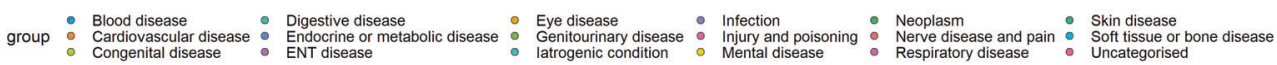

Fig. 2 Standardized mean differences in conditions among patients living with obesity compared to patients living without obesity in each database, stratified by COVID-19 cohort type (diagnosed and hospitalized). SMD $<0$ means the prevalence was greater in COVID-19 patients living without obesity, SMD $>0$ means the prevalence was greater in COVID-19 patients living with obesity. COVID-19 coronavirus disease 2019, CPRD Clinical Practice Research Datalink, CUIMC Columbia University Irving Medical Center, SIDIAP Information System for Research in Primary Care, SMD standardized mean difference, STARR-OMOP Stanford Medicine Research Data Repository, VA-OMOP United States Department of Veterans Affairs.

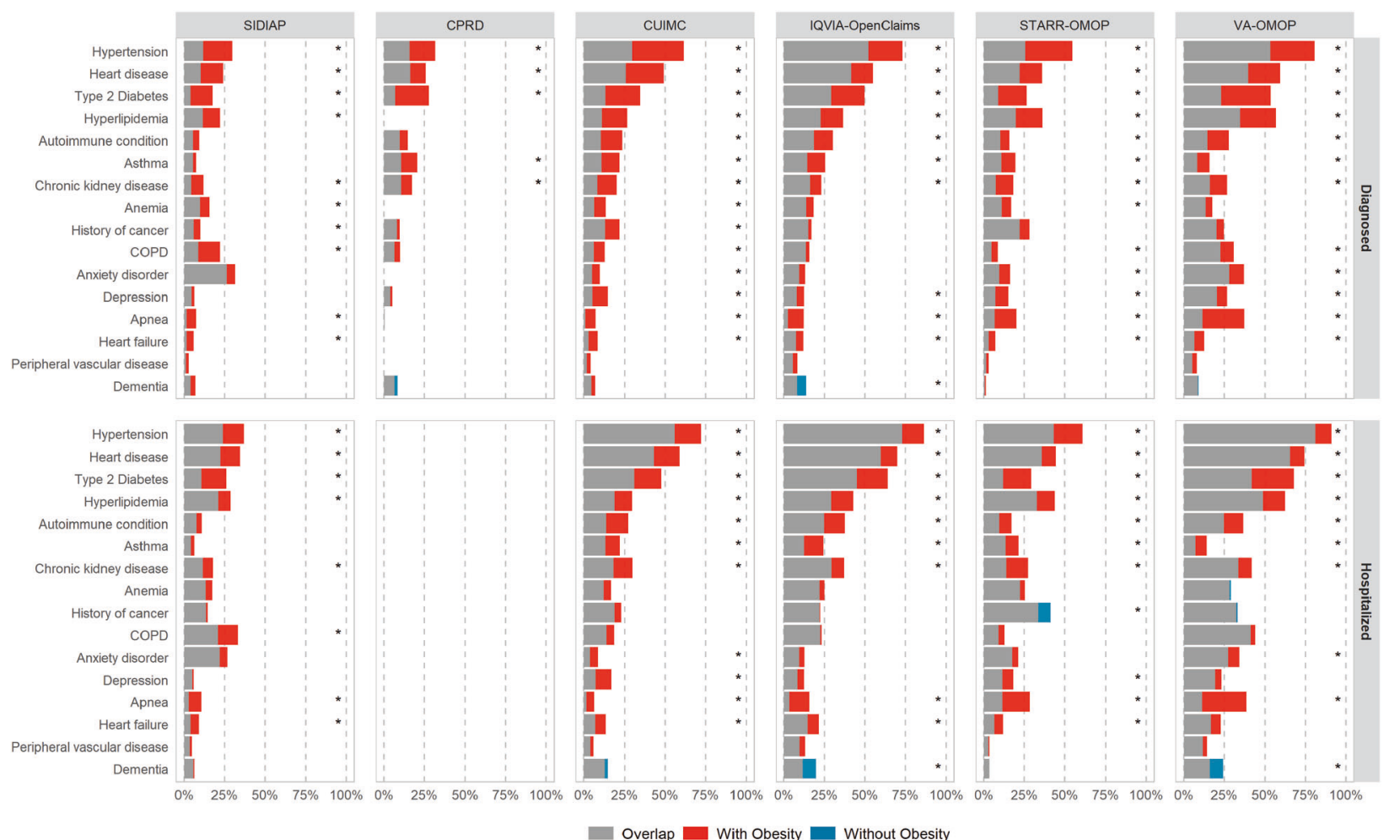

Fig. 3 Comorbidities at baseline among patients living with obesity compared to patients living without obesity in each database, stratified by COVID-19 cohort type (diagnosed and hospitalized). Prevalence of comorbidities for COVID-19 patients living with obesity (red) and without obesity (blue) are depicted in overlapped horizontal bars. The gray color is the overlap between groups. E.g., in CPRD, 32\% of COVID-19 patients living with obesity and 16\% living without obesity have hypertension. Comorbidities with a meaningful difference (|SMD|> 0.1 ) between patients living with and without obesity are marked with an asterisk $(*)$. COPD chronic obstructive pulmonary disease, COVID-19 coronavirus disease 2019, CPRD Clinical Practice Research Datalink, CUIMC Columbia University Irving Medical Center, SIDIAP Information System for Research in Primary Care, SMD standardized mean difference, STARR-OMOP Stanford Medicine Research Data Repository, VAOMOP United States Department of Veterans Affairs.

difference in the proportion of IS. Similarly, heart failure was also more frequent among PLWO than among patients without obesity in CUIMC: $7 \%$ vs $3 \%$, IQVIA-OpenClaims: $7 \%$ vs $5 \%$, STARR-OMOP: $16 \%$ vs $9 \%$, and VA-OMOP: $23 \%$ vs $17 \%$ ), these differences were meaningful in CUIMC and STARR-OMOP. Sepsis, cardiac arrhythmia, and cardiovascular disease events were slightly more frequent among PLWO, although SMDs were below 0.1 in all databases. Acute kidney injury was the only outcome that was more frequent among patients without obesity; however, this difference was not meaningful in any database. 

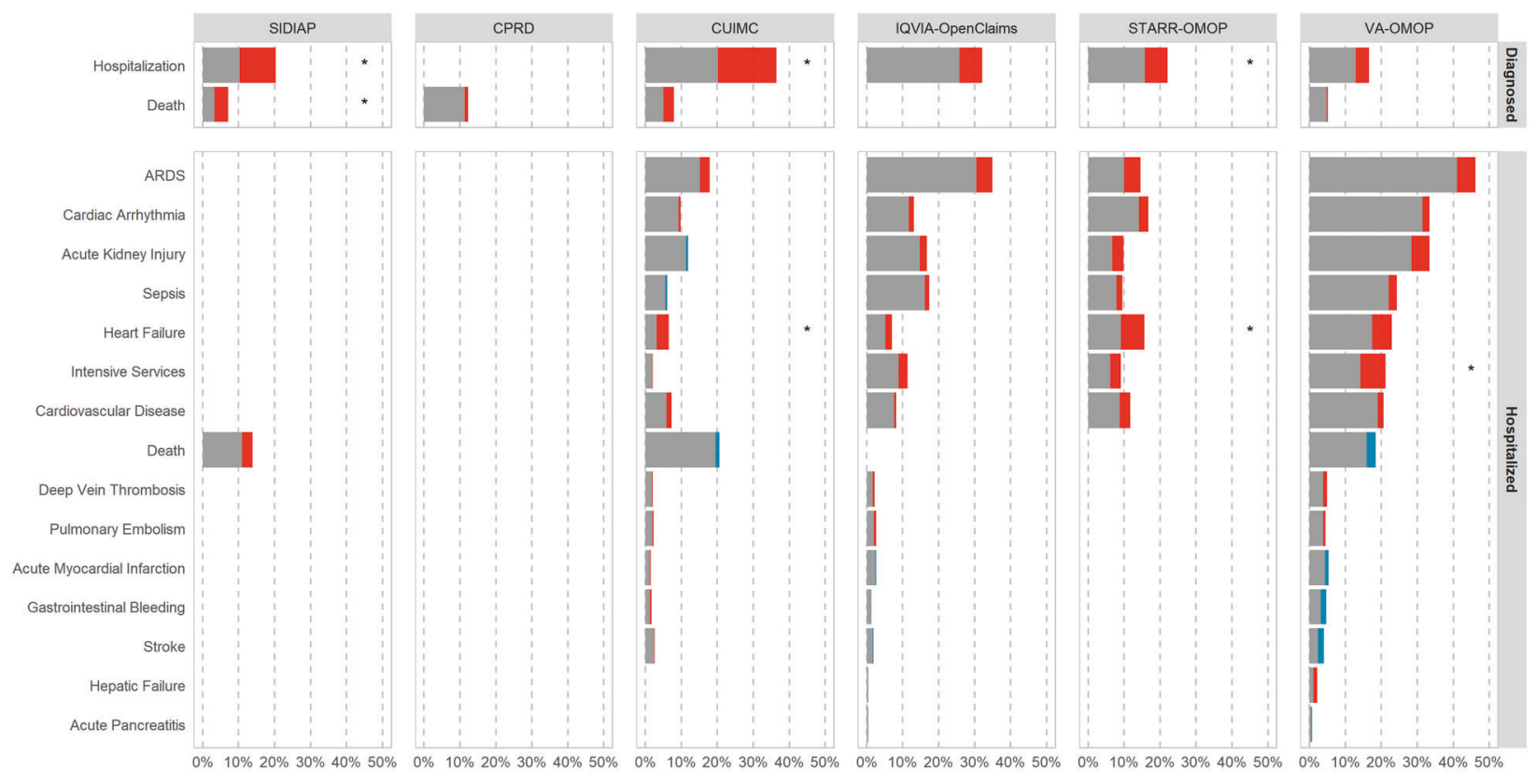

Overlap With Obesity Without Obesity

Fig. 4 A comparison of 30-day events among patients living with and without obesity in each database, by COVID-19 cohort type (diagnosed and hospitalized). Proportion of outcomes for COVID-19 patients living with obesity (red) and without obesity (blue) are depicted in overlapped horizontal bars. The gray color is the overlap between groups. E.g., in the diagnosed cohort, 20 and $10 \%$ of patients living with and without obesity in SIDIAP, respectively, were hospitalized. Outcomes with a meaningful difference $(|S M D|>0.1)$ between patients living with obesity and patients without obesity are marked with an asterisk (*). ARDS acute respiratory distress syndrome, COVID-19 coronavirus disease 2019, CPRD Clinical Practice Research Datalink, CUIMC Columbia University Irving Medical Center, SIDIAP Information System for Research in Primary Care, SMD standardized mean difference, STARR-OMOP Stanford Medicine Research Data Repository, VA-OMOP United States Department of Veterans Affairs.

As for fatality, there were no consistent nor meaningful differences between PLWO and patients without obesity in the hospitalized cohort: while it was higher for PLWO in SIDIAP $(14 \%$ vs $11 \%)$, there were no differences in CUIMC (20\% vs $21 \%)$ nor in VA-OMOP (16\% vs $18 \%)$.

\section{DISCUSSION}

In this large cohort study including 627044 COVID-19 patients from Spain, the UK, and the US, we found that the prevalence of obesity was higher among COVID-19 patients hospitalized (40\%) compared to those diagnosed (31\%). PLWO diagnosed and hospitalized with COVID-19 were more commonly female, and those hospitalized were younger than patients without obesity. The extraction of more than 15000 medical conditions revealed PLWO were not only more prone to have obesity-related comorbidities, such as hypertension, heart disease, and type 2 diabetes but also to more than a thousand different health conditions. After 30-days of follow-up, PLWO presented with higher hospitalization rates and intensive services requirements, although these differences were only meaningful in some databases.

Our study has several strengths, such as its large amount of data. By bringing together harmonized data using a federated approach, we have conducted a large-scale study while respecting the confidentiality of patient records. The international approach of this study is a strong asset given that we are investigating the intersection of two major global threats, namely the obesity epidemic and the COVID-19 pandemic. The former, together with the diverse healthcare settings and populations described in this study, increase the generalizability of our findings. Further, we provide a wide overview of the characteristics and outcomes of patients with and without obesity, using data visualization tools to summarize large amounts of medical data. This exhaustive characterization goes far beyond prior studies reporting few comorbidities and supports the generation of new hypotheses that can be tested in future studies. In addition, for the sake of transparency and reproducibility, we have made methods, tools, and all results publicly available. As CHARYBDIS is an ongoing study, results (included longer follow-up time) will be updated and new studies focussing on obesity could be conducted. All of the above has been accomplished through the coordinated efforts of the OHDSI community to provide a rapid response to the COVID19 pandemic.

Our study also has limitations. First, we cannot exclude a selection bias of COVID-19 cases due to underreporting in the context of testing restrictions and asymptomatic or paucisymptomatic cases that usually do not seek medical care. Additionally, testing policies have varied across countries and time depending on the course of the pandemic. Nevertheless, the inclusion of patients clinically diagnosed (not tested) in different settings likely provided consistency to our data, although it might have incurred in false positives. Second, we did not have information on BMI as a continuous variable, which prevented us from investigating the impact of different categories of obesity in COVID-19 outcomes. This might explain the higher proportion of comorbidities and outcomes observed in the US databases, as PLWO from the US might have higher BMls than those from Europe [20]. In addition, our definition of obesity included diagnoses and measurements recorded at any time prior to or at the index date, and therefore some individuals might have been misclassified due to changes in $\mathrm{BMI}$ since the most recently recorded status. However, previous evidence shows that BMI trajectories in adults are relatively stable, with a tendency to increase with age [21]. Therefore adults with obesity are likely to still have obesity over time. Finally, this study was underpinned by routinely collected data which can raise concerns about the quality of the data. Some databases are prone to oversampling certain groups of people as a result of how these 


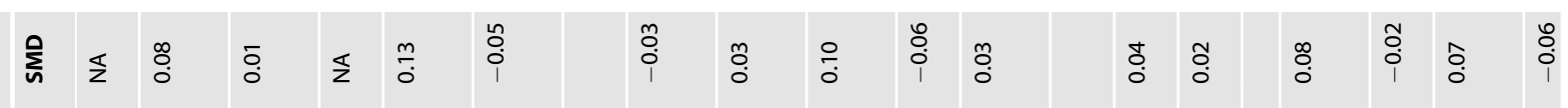

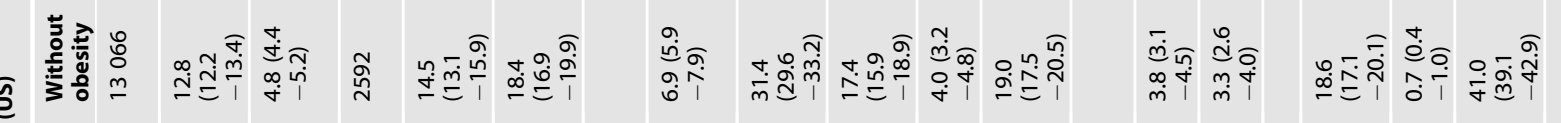

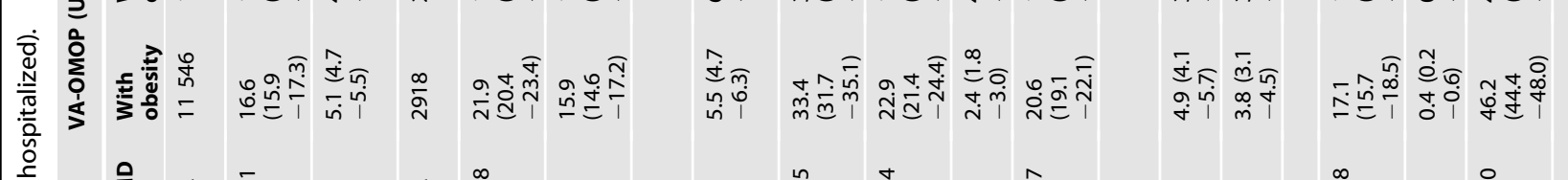

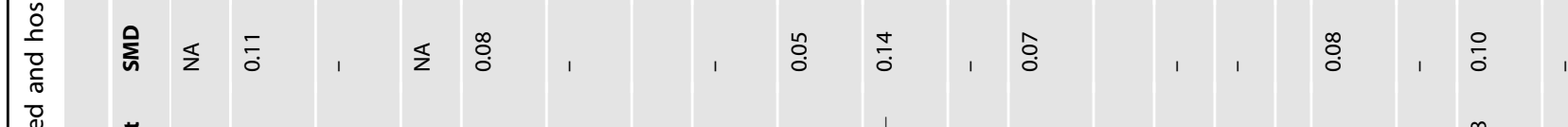

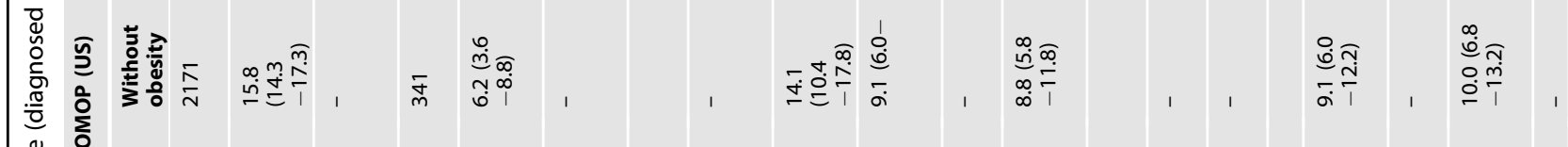

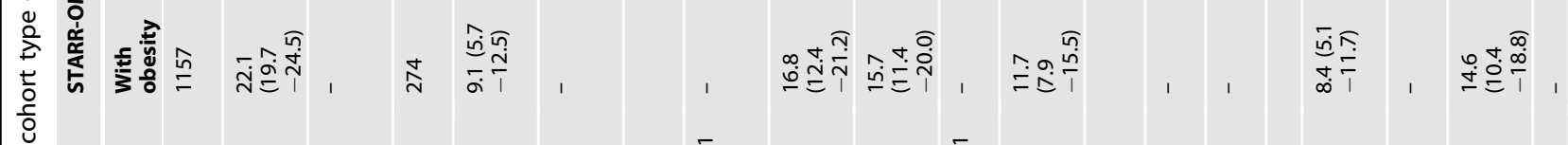

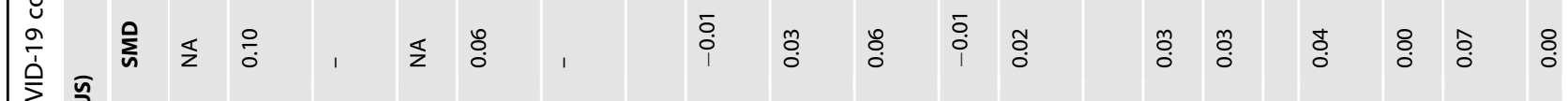

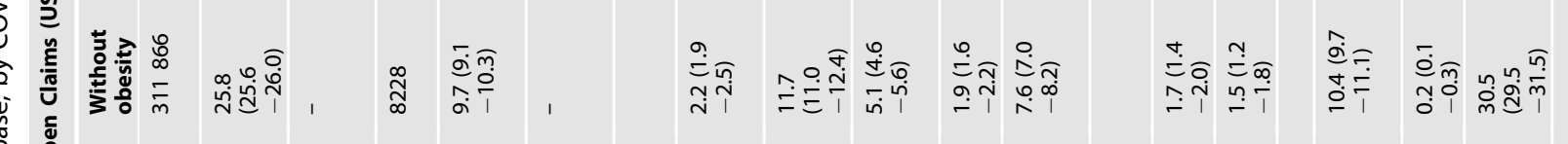

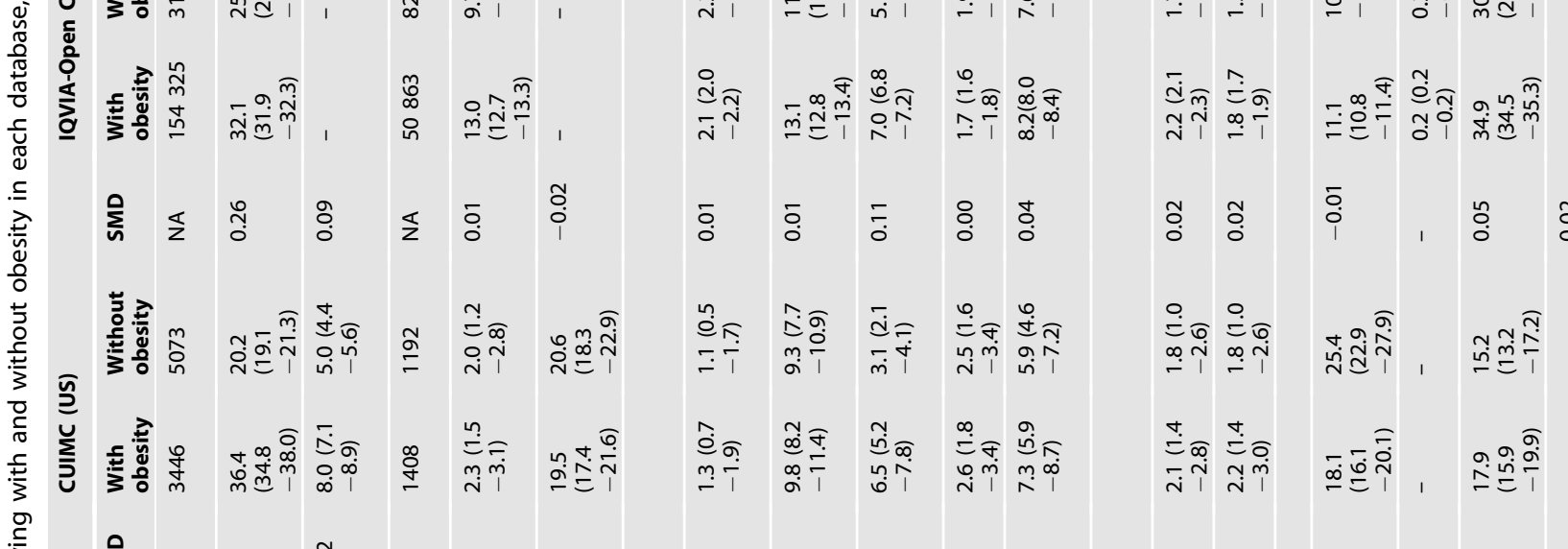

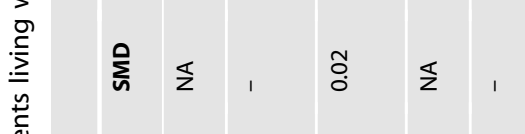

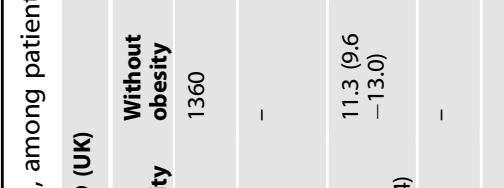

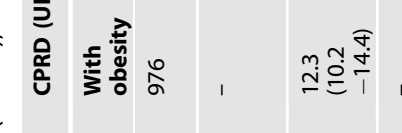

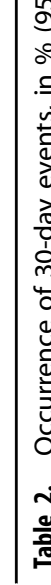

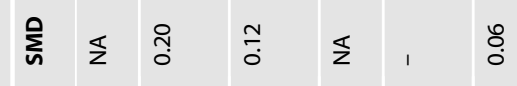

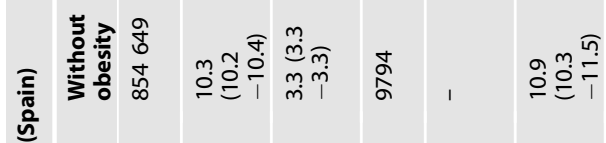

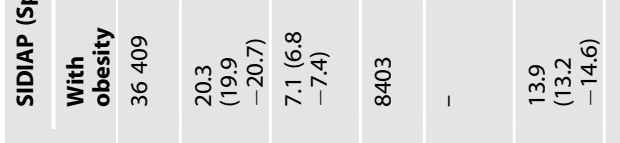

施 
data are captured (e.g., the Veterans Affairs system historically serves more men than women, routine claims data may only reflect health outcomes in commercially insured populations, etc.). Obesity, comorbidities, and outcomes were assessed based on having a record of a condition/measurement, therefore they may be underestimated. In addition, outcomes such as hospitalization or intensive services requirements are also influenced by factors external to the patient's condition (i.e., bed availability, criteria for admission), which might differ across databases. Even still, the consistency of our findings across databases that differ by setting and country lends credence to the generalizability of our findings.

Given the prevalence of obesity in Spain (24\%), the UK (27\%), and the US (37\%), a high proportion of PLWO among COVID-19 cases was expected [20]. However, the prevalence of obesity among diagnosed COVID-19 patients was higher than the general population in four databases: SIDIAP (Spain): 30\%; CPRD (UK): 42\%; CUIMC and VA-OMOP (US): 41 and 47\%, respectively, which is suggestive of an increased risk of diagnosis in PLWO. In addition, the prevalence of obesity was higher in hospitalized COVID-19 patients, with an overall prevalence of obesity of $40 \%$, which is in line with three cohort studies from the US that reported that 40 , 42 , and $48 \%$ of inpatients were living with obesity [17, 22, 23]. A large meta-analysis of observational studies reported that obesity is associated with a higher risk of testing positive for SARS-CoV-2 or being diagnosed with COVID-19 as well as of being hospitalized with COVID-19 [7]. While this could be due to an increased vulnerability to SARS-CoV-2 in PLWO, other hypotheses should be considered in future studies. On the one hand, individuals with obesity could be more likely to seek care and be tested for SARSCoV-2 since they are (presumably) a high-risk population, have multiple comorbidities, and are more prone to respiratory symptoms due to their compromised pulmonary function [2, 7]. On the other hand, given the fact that obesity disproportionately affects disadvantaged populations, potential differential exposures across subpopulation groups should also be explored (e.g., differential occupational risks) [2].

Women predominated among hospitalized patients with obesity, even though obesity rates are similar in both sexes in the three countries [20]. Although male sex is a well-established risk factor for COVID-19-related hospitalization and death, little is known about the role of obesity on COVID-19 outcomes stratified by sex $[14,23-25]$. Recent studies addressing this issue in secondary analyses have reported inconsistent results. A study conducted among UK Biobank participants found that the impact of BMI in COVID-19-related death was higher among females compared to men, while others have found a higher effect among males, opposite effects of sex in different age strata or null differences [26-29]. Thus, the intersection between sex/gender and obesity in relation to COVID-19-outcomes warrants further investigation. Because sex-stratification was beyond the prespecified analysis plan of our study, we were unable to report our results by sex, which could have provided valuable insights on the matter. We intend, however, to address this issue in upcoming studies from the CHARYBDIS project.

We also found that hospitalized PLWO were younger than those without obesity. Although younger individuals have less risk of infections and complications than older people due to having fewer comorbidities and a stronger immune system, this is not the case for those with obesity [2, 7, 30-32]. Some authors have postulated that PLWO younger than 60 years could have a greater risk of severe COVID-19 outcomes [33]. PLWO also had many more comorbidities than patients without obesity. Unsurprisingly, the highest differences were observed in obesity-related conditions, such as hypertension, diabetes, and heart disease, which have been identified as risk factors for severe COVID-19 outcomes $[14,17,25,34,35]$. However, as our findings revealed, PLWO with COVID-19 differ from patients without obesity in a wider range of medical conditions than previously described. Future etiological 
studies aiming to disentangle the effect of obesity in COVID-19 outcomes should have this information present and consider datadriven techniques to account for confounding, such as propensity score estimation and its adjustment methods [18].

Finally, PLWO experienced adverse events more frequently than those without obesity, particularly hospitalization and the requirement of intensive services. Certainly, our results must be interpreted carefully considering the differences in demographics and comorbidities between these groups. Interestingly, in patients hospitalized, we did not observe clear differences in fatality between patients with and without obesity. While two meta-analyses reported that obesity is associated with a higher risk of COVID-19 related mortality; other large observational studies from the US and the UK using finer categories of BMI only found an association with mortality for morbid obesity (BMls $\geq 35 \mathrm{~kg} / \mathrm{m}_{2}$ or $\geq 40 \mathrm{~kg} / \mathrm{m}_{2}$ ) $[7,25,28,29,34]$. Given the scarcity of evidence regarding the frequency of specific adverse events during hospitalization among PLWO, our findings are of special interest to the field and should be addressed in upcoming etiological studies.

In this large international cohort, we showed that among COVID-19 cases, PLWO were more likely to be female, have more comorbidities, and worse outcomes than patients without obesity. The prevalence of obesity was higher among hospitalized patients with COVID-19 compared to patients diagnosed with COVID-19. Our results may be useful in guiding clinical practice and aid future preventative strategies for patients living with obesity, as well as providing useful data to support subsequent etiological studies focussed on obesity and COVID-19.

\section{REFERENCES}

1. Di Angelantonio E, Bhupathiraju SN, Wormser D, Gao P, Kaptoge S, de Gonzalez $A B$, et al. Body-mass index and all-cause mortality: individual-participant-data meta-analysis of 239 prospective studies in four continents. Lancet. 2016;388:776-86.

2. Hu FB. Obesity Epidemiology. New York: Oxford University Press, 2009.

3. Moser JAS, Galindo-Fraga A, Ortiz-Hernández AA, Gu W, Hunsberger S, GalánHerrera JF. et al.Underweight, overweight, and obesity as independent risk factors for hospitalization in adults and children from influenza and other respiratory viruses.Influenza Other Respir Viruses.2019;13:3-9.

4. WHO. WHO Director-General's opening remarks at the media briefing on COVID19-11 March 2020. WHO. 2020. https://www.who.int/directorgeneral/speeches/ detail/who-director-general-s-opening-remarks-at-the-media-briefing-oncovid19---11-march-2020.

5. Louie JK, Acosta M, Samuel MC, Schechter R, Vugia DJ, Harriman K, et al. A novel risk factor for a novel virus: obesity and 2009 pandemic influenza a (H1N1). Clin Infect Dis. 2011;52:301-12.

6. GOV.UK. New obesity strategy unveiled as country urged to lose weight to beat coronavirus (COVID-19) and protect the NHS. GOV.UK. 2020. https://www.gov.uk/ government/news/new-obesity-strategy-unveiled-as-country-urged-to-loseweight-to-beat-coronavirus-covid-19-and-protect-the-nhs.

7. Popkin BM, Du S, Green WD, Beck MA, Algaith T, Herbst CH, et al. Individuals with obesity and COVID-19: a global perspective on the epidemiology and biological relationships. Obes Rev. 2020;21:e13128.

8. Griffith GJ, Morris TT, Tudball MJ, Herbert A, Mancano G, Pike L, et al. Collider bias undermines our understanding of COVID-19 disease risk and severity. Nat Commun. 2020;11:1-12.

9. Voss EA, Makadia R, Matcho A, Ma Q, Knoll C, Schuemie M, et al. Feasibility and utility of applications of the common data model to multiple, disparate observational health databases. J Am Med Informatics Assoc. 2015;22:553-64.

10. Hripcsak G, Duke JD, Shah NH, Reich CG, Huser V, Schuemie MJ, et al. Observational Health Data Sciences and Informatics (OHDSI): opportunities for observational researchers. Stud Health Technol Inform. 2015;216:574-8.

11. Bolíbar B, Fina Avilés F, Morros R, Del Mar Garcia-Gil M, Hermosilla E, Ramos R, et al. Base de datos SIDIAP: La historia clínica informatizada de Atención Primaria como fuente de información para la investigación epidemiológica. Med Clin. 2012;138:617-21.

12. Herrett E, Gallagher AM, Bhaskaran K, Forbes H, Mathur R, Staa Tvan, et al. Data resource profile: Clinical Practice Research Datalink (CPRD). Int J Epidemiol. 2015;44:827-36.

13. Datta S, Posada J, Olson G, Li W, O'Reilly C, Balraj D, et al. A new paradigm for accelerating clinical data science at Stanford Medicine. arXiv:2003.10534v1
[Preprint]. 2020 [cited 2020 Aug 20]. Available from: https://arxiv.org/abs/ 2003.10534v1

14. Burn E, Tebé C, Fernandez-Bertolin S, Aragon M, Recalde M, Roel E, et al. The natural history of symptomatic COVID-19 during the first wave in Catalonia. Nat Commun. 2021;12:1-12.

15. Burn E, You SC, Sena AG, Kostka K, Abedtash H, Abrahão MTF, et al. Deep phenotyping of 34,128 adult patients hospitalised with COVID-19 in an international network study. Nat Commun. 2020;11:5009.

16. Observational Health Data Sciences and Informatics. The Book of OHDSI (Independently published). 2019. https://ohdsi.github.io/TheBookOfOhdsi/.

17. Richardson S, Hirsch JS, Narasimhan M, Crawford JM, McGinn T, Davidson KW, et al. Presenting characteristics, comorbidities, and outcomes among 5700 patients hospitalized with COVID-19 in the New York City area. JAMA. 2020;323:2052-9.

18. Austin PC. An introduction to propensity score methods for reducing the effects of confounding in observational studies. Multivariate Behav Res. 2011;46: 399-424.

19. Austin PC. Using the standardized difference to compare the prevalence of a binary variable between two groups in observational research. Commun Stat Simul Comput. 2009;38:1228-34.

20. World Health Organization. WHO Overweight and obesity. World Health Organization. 2020. https://www.who.int/data/gho/data/themes/topics/topic-details/ $\mathrm{GHO} /$ ncd-risk-factors.

21. Kelly SP, Lennon H, Sperrin M, Matthews C, Freedman ND, Albanes D, et al. Body mass index trajectories across adulthood and smoking in relation to prostate cancer risks: the NIH-AARP diet and health study. Int J Epidemiol. 2019;48:464-73.

22. Garg S, Kim L, Whitaker M, O'Halloran A, Cummings C, Holstein R, et al. Hospitalization rates and characteristics of patients hospitalized with laboratoryconfirmed coronavirus disease 2019-COVID-NET, 14 states, March 1-30, 2020. MMWR Morb Mortal Wkly Rep. 2020;69:458-64.

23. Petrilli CM, Jones SA, Yang J, Rajagopalan H, O'Donnell L, Chernyak Y, et al. Factors associated with hospital admission and critical illness among 5279 people with coronavirus disease 2019 in New York City: prospective cohort study. BMJ. 2020;369:m1966.

24. Docherty $A B$, Harrison EM, Green CA, Hardwick HE, Pius R, Norman L, et al. Features of 20133 UK patients in hospital with covid-19 using the ISARIC WHO Clinical Characterisation Protocol: prospective observational cohort study. BMJ. 2020;369:m1985.

25. Gupta S, Hayek SS, Wang W, Chan L, Mathews KS, Melamed ML, et al. Factors associated with death in critically III patients with coronavirus disease 2019 in the US. JAMA Intern Med. 2020;180:1436-47.

26. Anderson MR, Geleris J, Anderson DR, Zucker J, Nobel YR, Freedberg D, et al. Body mass index and risk for intubation or death in SARS-CoV-2 infection: a retrospective cohort study. Ann Intern Med. 2020;173:782-90.

27. Sattar N, Ho FK, Gill JM, Ghouri N, Gray SR, Celis-Morales CA, et al. BMI and future risk for COVID-19 infection and death across sex, age and ethnicity: preliminary findings from UK biobank. Diabetes Metab Syndr Clin Res Rev. 2020;14:1149-51.

28. Tartof SY, Qian L, Hong V, Wei R, Nadjafi RF, Fischer H, et al. Obesity and mortality among patients diagnosed with COVID-19: results from an integrated health care organization. Ann Intern Med. 2020;173:773-81.

29. Du Y, Lv Y, Zha W, Zhou N, Hong X. Association of body mass index (BMI) with critical COVID-19 and in-hospital mortality: a dose-response meta-analysis. Metabolism. 2021;117:154373.

30. Divo MJ, Martinez $\mathrm{CH}$, Mannino DM. Ageing and the epidemiology of multimorbidity. Eur Respir J. 2014;44:1055-68.

31. Simon AK, Hollander GA, McMichael A. Evolution of the immune system in humans from infancy to old age. Proc R Soc B Biol Sci. 2015;282:20143085.

32. Muscogiuri G, Pugliese G, Laudisio D, Castellucci B, Barrea L, Savastano S, et al. The impact of obesity on immune response to infection: plausible mechanisms and outcomes. Obes Rev. 2021;22:e13216.

33. Lighter J, Phillips M, Hochman S, Sterling S, Johnson D, Francois F, et al. Obesity in patients younger than 60 years is a risk factor for COVID-19 hospital admission. Clin Infect Dis. 2020;71:896-7.

34. Williamson EJ, Walker AJ, Bhaskaran K, Bacon S, Bates C, Morton CE, et al. Factors associated with COVID-19-related death using OpenSAFELY. Nature. 2020;584:430-6.

35. Reilev M, Kristensen KB, Pottegård A, Lund LC, Hallas J, Ernst MT, et al. Characteristics and predictors of hospitalization and death in the first 11122 cases with a positive RT-PCR test for SARS-CoV-2 in Denmark: a nationwide cohort. Int J Epidemiol. 2020;49:1468-81.

\section{ACKNOWLEDGEMENTS}

We would like to acknowledge the patients who suffered from or died of this devastating disease, and their families and carers. We would also like to thank the 
healthcare professionals involved in the management of COVID-19 during these challenging times, from primary care to intensive care units.

\section{AUTHOR CONTRIBUTIONS}

MR, ER, APU, PR, DPA, KK, and TDS conceived and designed the study. SLD, TF, KEL, MEM, KN, JDP, CGR, NHS, PR, KK, and TDS coordinated data contributions at their respective sites. AP, AGS, TF, SFB, JDP, KK, and TDS analyzed the data; MR, ER, and AP produced the figures and tables. MR, ER, EB, DRM, FN, PR, LMS, DPA, KK, and TDS interpreted the data. MR, ER, and TDS searched the literature and wrote the first draft with insightful contributions from EB, LYHL, JCEL, DRM, FN, PR, LMS, DPA, and KK. All authors contributed to the revision of the first draft, reviewed and approved the final version of the paper.

\section{FUNDING}

The European Health Data \& Evidence Network has received funding from the Innovative Medicines Initiative 2 Joint Undertaking (JU) under grant agreement No 806968. The JU receives support from the European Union's Horizon 2020 research and innovation program and EFPIA. This research received partial support from the National Institute for Health Research (NIHR) Oxford Biomedical Research Center (BRC), US National Institutes of Health, US Department of Veterans Affairs, Janssen Research \& Development, and IQVIA. The University of Oxford received funding related to this work from the Bill \& Melinda Gates Foundation (Investment ID INV016201 and INV-019257). APU has received funding from the Medical Research Council (MRC) [MR/K501256/1, MR/N013468/1] and Fundación Alfonso Martín Escudero (FAME) (APU). VINCI [VA HSR RES 13-457] (SLD, MEM, KEL). JCEL has received funding from the Medical Research Council (MR/K501256/1) and Versus Arthritis (21605). MR is funded by Wereld Kanker Onderzoek Fonds (WKOF), as part of the World Cancer Research Fund International grant program [grant number: 2017/ 1630]. No funders had a direct role in this study. The views and opinions expressed are those of the authors and do not necessarily reflect those of the Clinician Scientist Award program, NIHR, Department of Veterans Affairs or the United States Government, NHS, or the Department of Health, England.

\section{COMPETING INTERESTS}

All authors have completed the ICMJE uniform disclosure form at www.icmje.org/ coi_disclosure.pdf and declare: Mr. Sena reports personal fees from Janssen Research \& Development, outside the submitted work; Dr. DuVall reports grants from Anolinx, LLC, grants from Astellas Pharma, Inc, grants from AstraZeneca Pharmaceuticals LP, grants from Boehringer Ingelheim International $\mathrm{GmbH}$, grants from Celgene Corporation, grants from Eli Lilly and Company, grants from Genentech Inc., grants from Genomic Health, Inc., grants from Gilead Sciences Inc., grants from GlaxoSmithKline PLC, grants from Innocrin Pharmaceuticals Inc., grants from Janssen Pharmaceuticals, Inc., grants from Kantar Health, grants from Myriad Genetic Laboratories, Inc., grants from Novartis International AG, grants from Parexel International Corporation through the University of Utah or Western Institute for Biomedical Research, outside the submitted work; Mr Ahmed reports funding from the NIHR Oxford Biomedical Research Center (BRC), Aziz Foundation, Wolfson Foundation, and the Royal College Surgeons of England; Dr. Golozar reports personal fees from Regeneron Pharmaceuticals, outside the submitted work. She is a full-time employee at Regeneron Pharmaceuticals. This work was not conducted at Regeneron Pharmaceuticals. Miss Lane is supported by a Medical Research Council Doctoral Research Fellowship (MR/K501256/1) and a Versus Arthritis Clinical Research Fellowship (21605). Dr. Morales is supported by a Wellcome Trust Clinical Research Development Fellowship (Grant 214588/Z/18/Z) and reports grants from Chief Scientist Office (CSO), grants from Health Data Research UK (HDR-UK), grants from National Institute of Health Research (NIHR), outside the submitted work; Dr. Nyberg reports other from AstraZeneca, outside the submitted work; Dr. Subbian reports grants from National Science Foundation, grants from State of Arizona; Arizona Board of Regents, grants from Agency for Healthcare Research and Quality, outside the submitted work; Dr Prieto-Alhambra reports grants and other from AMGEN; grants, non-financial support and other from UCB Biopharma; grants from Les Laboratoires Servier, outside the submitted work; and Janssen, on behalf of IMI-funded EHDEN and EMIF consortiums, and Synapse Management Partners have supported training programs organized by DPA's department and open for external participants. Ms. Kostka and Dr. Reich report being employees of IQVIA Inc at the time the analysis was conducted. Dr. Rijnbeek reports grants from Innovative Medicines Initiative, grants from Janssen Research and Development, during the conduct of the study. Dr. Ryan is an employee of Janssen Research and Development and shareholder of Johnson \& Johnson. The views expressed are those of the authors and do not necessarily represent the views or policy of the Department of Veterans Affairs or the United States Government. No other relationships or activities that could appear to have influenced the submitted work.

\section{ETHICAL APPROVAL}

All the data partners received Institutional Review Board (IRB) approval or exemption. STARR-OMOP had approval from IRB Panel \#8 (RB-53248) registered to Leland Stanford Junior University under the Stanford Human Research Protection Program (HRPP). The use of VA data was reviewed by the Department of Veterans Affairs Central IRB, was determined to meet the criteria for exemption under Exemption Category 4 (3), and approved for Waiver of HIPAA Authorization. The research was approved by the Columbia University Institutional Review Board as an OHDSI network study. The use of SIDIAP was approved by the Clinical Research Ethics Committee of the IDIAPJGol (project code: 20/070-PCV). The use of CPRD was approved by the Independent Scientific Advisory Committee (ISAC) (protocol number 20_059RA2). The use of IQVIA-OpenClaims was exempted from IRB approval.

\section{TRANSPARENCY DECLARATION}

Lead authors affirm that the paper is an honest, accurate, and transparent account of the study being reported; that no important aspects of the study have been omitted; and that any discrepancies from the study as planned have been explained.

\section{DATA SHARING STATEMENT}

Analyses were performed locally in compliance with all applicable data privacy laws. Although the underlying data is not readily available to be shared, authors contributing to this paper have direct access to the data sources used in this study. All results (e.g., aggregate statistics, not presented at a patient-level with redactions for minimum cell count) are available for public inquiry. These results are inclusive of site-identifiers by contributing data sources to enable interrogation of each contributing site. All analytic code and result sets are made available at: https:// github.com/ohdsi-studies/Covid19CharacterizationCharybdis.

\section{ADDITIONAL INFORMATION}

Supplementary information The online version contains supplementary material available at https://doi.org/10.1038/s41366-021-00893-4.

Correspondence and requests for materials should be addressed to T.D-S.

Reprints and permission information is available at http://www.nature.com/ reprints

Publisher's note Springer Nature remains neutral with regard to jurisdictional claims in published maps and institutional affiliations.

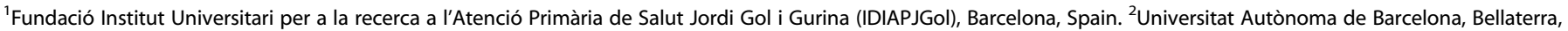

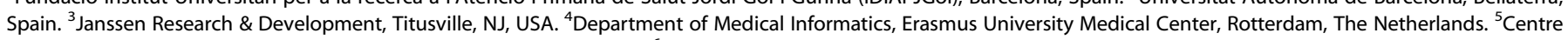

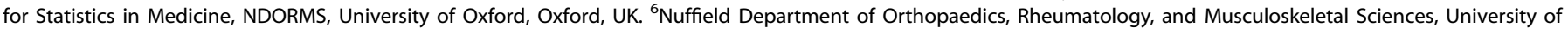

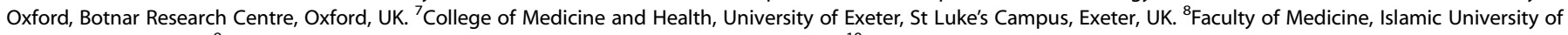

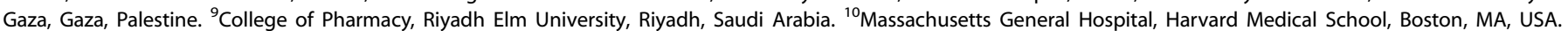

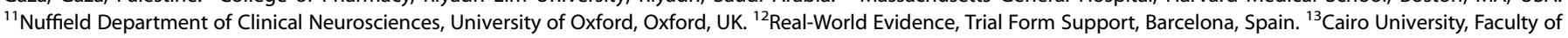

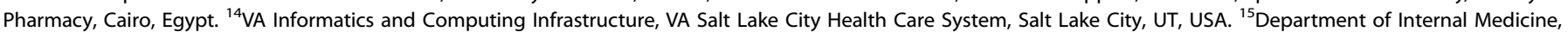

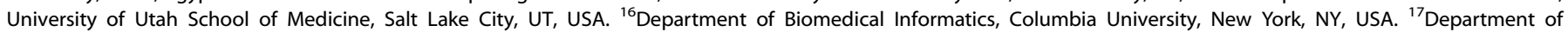

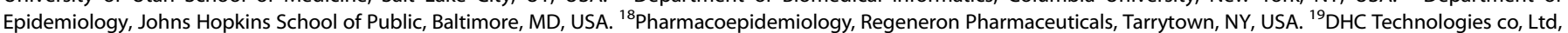

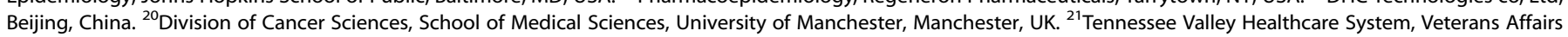

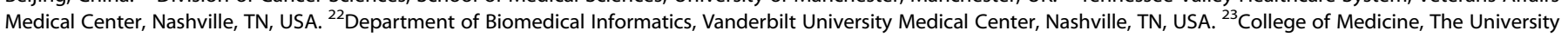


of Arizona, Tucson, AZ, USA. ${ }^{24}$ Division of Population Health and Genomics, University of Dundee, Dundee, UK. ${ }^{25}$ New York-Presbyterian Hospital, New York, NY, USA. ${ }^{26}$ School of Public Health and Community Medicine, Institute of Medicine, Sahlgrenska Academy, University of Gothenburg, Gothenburg, Sweden. ${ }^{27}$ Department of Medicine, Stanford University, Palo Alto, CA, USA. ${ }^{28}$ Real World Solutions, IQVIA, Cambridge, MA, USA. ${ }^{29}$ Data Science to Patient Value Program, Department of Medicine, University of Colorado Anschutz Medical Campus, Aurora, CO, USA. ${ }^{30}$ College of Engineering, The University of Arizona, Tucson, AZ, USA. ${ }^{31}$ School of Population Medicine and Public Health, Chinese Academy of Medical Sciences \& Peking Union Medical College, Beijing, China. ${ }^{32}$ Melbourne School of Population and Global Health, The University of Melbourne, Melbourne, VIC, Australia. ${ }^{33}$ Institute of Health Management, Southern Medical University, Guangzhou, China. ${ }^{34}$ Nanfang Hospital, Southern Medical University, Guangzhou, China. ${ }^{35}$ The OHDSI Center at the Roux Institute, Northeastern University, Portland, ME, USA. ${ }^{37}$ These authors contributed equally: Martina Recalde, Elena Roel. ${ }^{37}$ These authors jointly supervised this work: Kristin Kostka, Talita Duarte-Salles. ${ }^{\bowtie}$ email: tduarte@idiapjgol.org

(i) Open Access This article is licensed under a Creative Commons Attribution 4.0 International License, which permits use, sharing, adaptation, distribution and reproduction in any medium or format, as long as you give appropriate credit to the original author(s) and the source, provide a link to the Creative Commons license, and indicate if changes were made. The images or other third party material in this article are included in the article's Creative Commons license, unless indicated otherwise in a credit line to the material. If material is not included in the article's Creative Commons license and your intended use is not permitted by statutory regulation or exceeds the permitted use, you will need to obtain permission directly from the copyright holder. To view a copy of this license, visit http://creativecommons. org/licenses/by/4.0/.

(c) The Author(s) 2021 